\title{
Morphofunctional Changes of the Kidneys in Non-Tumour Cholestasis
}

\author{
Kashaeva M.D.* Gavrilova K.V. Dyukov D.S. Proshin A.V. Proshina L.G.
}

\author{
Golushko V.A.
}

\begin{abstract}
Institute of medical education of Yaroslav-the-Wise Novgorod State University the city of Veliky Novgorod, Russia *Corresponding author. Email: kashaevamrd@mail.ru
\end{abstract}

\begin{abstract}
The morphofunctional changes of kidneys at cholestasis of various duration in 170 patients suffered from obstructive jaundice of non-neoplastic aetiology were analyzed. Taking part in the processes of the metabolism of biologically active substances, including hormones, the liver has a regulatory effect on many kidney functions, including filtration and resorption. It was revealed that the depth of homeostatic disorders is directly dependent on the duration of cholestasis and the level of bilirubinemia in biliary obstruction, leading to significant functional and structural lesions of the kidneys. Thus, in the case of cholestasis lasting up to 10 days, there was a moderate decrease in diuresis without renal dysfunction, with 2 weeks of jaundice, both a decrease in diuresis and a moderate decrease in glomerular filtration were detected, and a fluctuation within the subnormal values of tubular reabsorption, with cholestasis 3-6 weeks, along with a pronounced decrease in diuresis, the functional ability of the kidneys were reduced. It was proved that the conducted complex conservative therapy led to stabilization of the function of the kidneys and facilitate better tolerance to the operations caused by the improving of the liver function.
\end{abstract}

\section{Keywords: duration of cholestasis, homeostatic disorders, structural changes of the liver and kidneys,} preoperative preparation, the functional ability of the kidneys.

\section{INTRODUCTION}

In case of cholestasis, there is an increase in endogenous intoxication, a disruption of the water-electrolyte balance, leading to a disorder of both general and hepatic and renal hemodynamics, and, consequently, to renal hypoxia [1-3]. In this case, the disruption of the tubular and glomerular systems with a decrease in the excretion of sodium in the urine and an increase in potassium occurs. The development of resistant ascites with a decrease in the volume of circulating plasma is promoted by diuretics, especially contrasted with a decrease in oncotic pressure as a consequence of dysproteinemia. Unsystematic long-term taking of diuretics leads to potassium losses and a decrease in sodium excretion in the urine, and, consequently, to disruption of the absorption and reabsorption of the kidneys $[3,4,6]$

An important role in the pathogenesis of hepatorenal syndrome (HRS) is played by the tubular sodium reabsorption, portal hypertension (PHT), the decrease of angiotensinogen and $\alpha_{2}$-globulin in the liver, the increased absorption of endotoxins and gram-negative bacteria from the digestive tract. The administering diuretics stimulating the renin-angiotensin system with the development of hypovolemia in this case and the administering nonsteroidal anti-inflammatory drugs suppressing the synthesis of renal prostaglandins cause the afferent vasoconstriction with a decrease and redistribution of renal blood flow, the hyperperfusion of the renal cortex ordinary with combination of the renal intravascular coagulation $[5,8,9]$. These disruptions quickly lead to a decrease in filtration function. Because of this, the progressive uremia increases hepatic cell and hepatic-cerebral insufficiency due to the additional formation of nitrogenous slags in the intestine and the suppression of hepatocytes regenerating. Diuretics removing potassium from the body cause the development of hypokalemia and metabolic alkalosis, that contributes to the absorption and the transport of ammonium ions in the intestine and the increase of kidney and liver cells damage $[10,11]$.

Hepatotoxic substances leading to severe functional and structural changes in the liver are accompanied by a disruption of the filtration and reabsorption functions of the kidneys, and even by structural damage of various severity of the glomerular apparatus.

Disruption of intrahepatic hemodynamics leads to a significant change in renal blood flow, to hypoxia of the cortical substance, which causes the disorders of the glomerular apparatus, dystrophic changes in the tubules and the impairment of the penetration of cell membranes [4-6].

The mechanism of kidney damage in cholestasis is largely due to the toxic effect of conjugated bilirubin released through the glomerular membrane. Being closely related to plasma albumin, unconjugated bilirubin is 
practically not filtered. It should be noted that the kidneys, which determine the high activity of glucuronyltransferase, participate in extrahepatic binding of bilirubin. Thus, unconjugated bilirubin is not filtered, but it accumulates in large quantities in the interstitium, causing staining of the renal papillae. Accumulation of bilirubin destroys the papillary zone and disrupts the function of the tubules and interstitium [12-14]. First of all, it is evidenced by the disruption of urine concentration and the reabsorption of water and formation of free water $[3,12,15]$. In the case of toxic and hemodynamic disorders in the kidneys with the accumulation of bilirubin due to biliary obstruction, the great importance is attached to the altered renal perfusion and the increased susceptibility to hypovolemic shock. For a long time hyperbilirubinemia as well as the surgery on the biliary tract caused by obstructive jaundice have been considered one of the main causes of hepatorenal syndrome. Obstructive jaundice with rapidly increased bilirubin and the decompression surgery lead to the development of renal failure, and in some cases, to an irreversible hepatorenal syndrome $[1,3,4,13]$.

The mortality rate in the group of patients with acute renal failure in the case of cholestasis is $57 \%$. The frequency of deaths correlates with the level of hyperbilirubinemia. Thus, when the content of bilirubin in serum was over $342 \mathrm{mmol} / \mathrm{l}$, the mortality rate was $85 \%$, and below $171 \mathrm{mmol} / \mathrm{l}-33 \%$ [4-6]. Thus, in hyperbilirubinemia, the severe degree of endotoxicosis, the hemodynamic disorders, as well as the direct effect of bilirubin on the renal tissue, causing severe local toxic damage are registered. An important role in the outcomes of obstructive jaundice is played by the development of endogenous intoxication syndrome, which is the main factor composing the clinical findings of the disease and determining the nature and outcome of it. High mortality in obstructive jaundice of non-tumour aetiology is explained by the discrepancy between the functional capabilities of the liver and the severity of the surgeries $[8$, $11,12]$. The crucial role of endogenous intoxication in the pathogenesis of obstructive jaundice of non-tumour aetiology determines the importance of just-in-time intensive complex therapy, which is capable to prevent the development of irreversible impairments of the structure and function of many organs and systems and contributing to the repair and adaptation processes [15].

\section{PURPOSE OF RESEARCH}

The purpose of the study is to analyze the renal dysfunction depending on the duration of cholestasis and the methods of preoperative preparation.

\section{METHODS AND MATERIALS}

The study includes the analysis of clinical data of 170 patients who were treated for cholestasis of non-tumour aetiology in the Novgorod Regional Clinical Hospital
(NRCH) and the Central Clinical Hospital of Veliky Novgorod (CCHN). The age of patients ranged from 16 to 70 years. The patients over 60 years of age accounted for $61.7 \%$. Males were $22.1 \%$ and females $-77.9 \%$. The most common cause of jaundice was choledocholithiasis (73.5\%), among the others - cicatricial strictures of the choledochus $(9.7 \%)$, chronic pancreatitis $(9.7 \%)$, pericholedocheal lymphadenitis $(5.2 \%)$, stenosis of the major duodenal papilla - MDP (1.9\%). Among 170 patients, 71 were admitted with obstructive jaundice lasting up to 10 days, 56 patients - up to 2 weeks, 43 ones from 3 to 6 weeks. Depending on the range of preoperative preparation, the patients were divided into 2 groups. The first group (the control group) - 90 patients (respectively, the duration of cholestasis $-27,43,20$ ). They are operated urgently after short-term traditional preparation within 2-8 hours. The second group (the main group) was counted 80 patients (respectively $-45,13,22$ ). They were operated after complex multicomponent preoperative preparation within 3-7 days. All patients underwent the clinical, biochemical studies, also the general analysis of urine was determined, and the sample of Rehberg-Tareev was conducted. The results obtained in the course of the studies were subjected to methods of parametric and nonparametric statistics.

\section{RESULTS}

In the case of cholestasis lasting for 10 days, there was a moderate decrease in diuresis without renal dysfunction, but in the case of 2-week jaundice, both a decrease in diuresis and a moderate decrease in glomerular filtration and fluctuation within the normal values of tubular reabsorption were revealed. In patients admitted late (3-6 weeks), along with a marked decrease in diuresis, the functional ability of the kidneys was generally reduced (table. 1).

After conservative therapy for jaundice lasting up to 10 days, the daily diuresis, although reduced, did not decrease in comparison with the initial one, the glomerular filtration and tubular reabsorption were within normal values. In case of jaundice lasting up to 2 weeks, there was no further decrease in diuresis, the glomerular filtration was at the lower limit of the norm, the tubular reabsorption in comparison with 10-day jaundice was moderately reduced with fluctuations from 98.2 to $99 \%$ (table. 1). In patients with prolonged jaundice (3-6 weeks) the daily diuresis was reduced, the glomerular filtration and tubular reabsorption impaired, but they did not deteriorate, even a few, although not significantly improved. Thus, the conservative therapy led to stabilization of the kidney function, which contributed to better tolerability of operations on the background of the improving liver function.

The comparative analysis of the functional parameters of the kidneys after surgical treatment before discharge in the first and second groups is of great interest. As can be seen from table 2, the complete normalization of renal function by the time of discharge after radical surgery and 
restoration of bile passage occurs in patients with 10-day jaundice, receiving 3-7 days of preoperative preparation.

Table 1 The state of renal function after 3-7 days of preoperative preparation in obstructive jaundice (M $\pm m, R)$

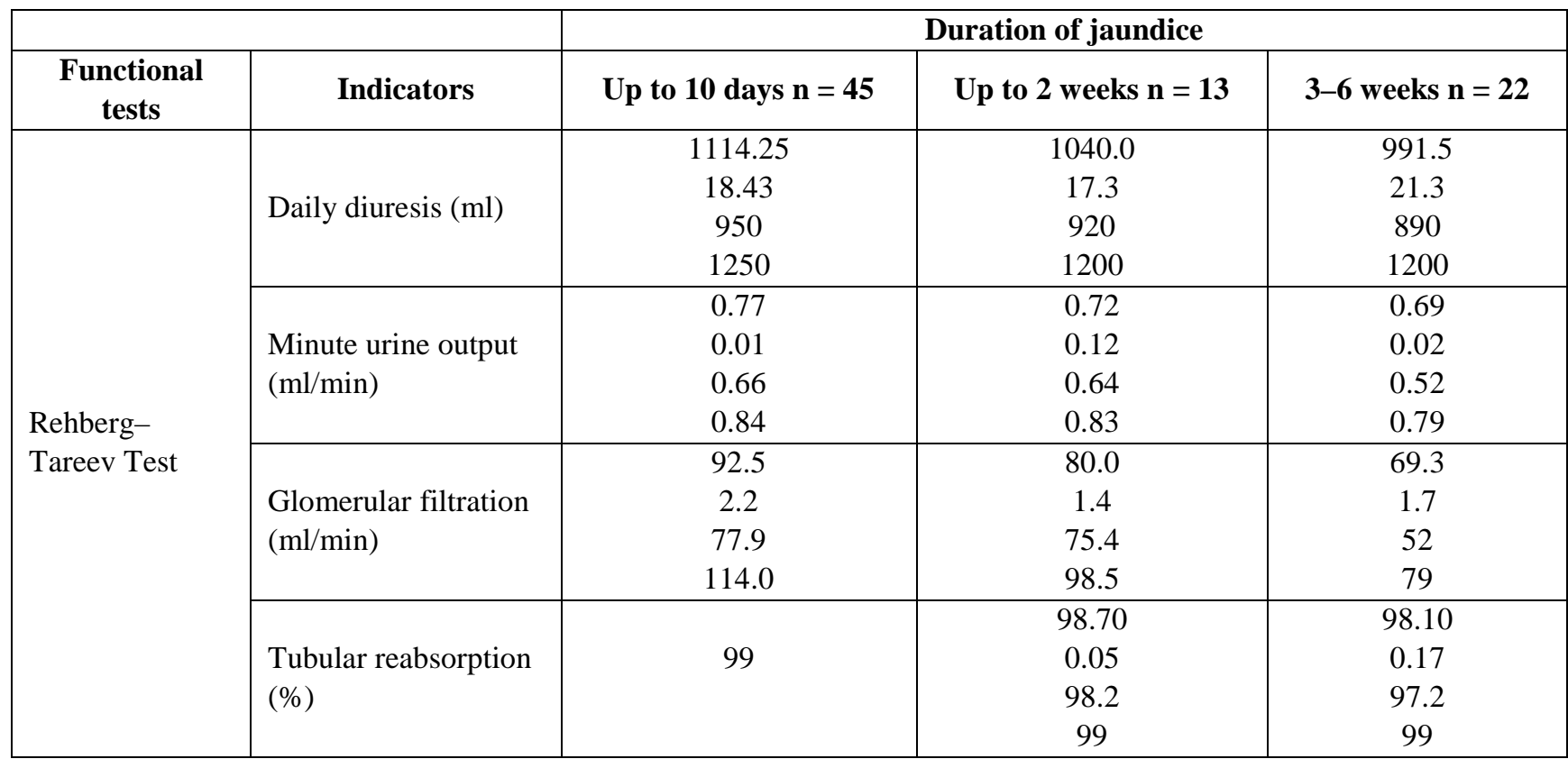

Table 2 Renal function after the surgery without preoperative preparation before the discharge $(\mathrm{M} \pm \mathrm{m} ; \mathrm{R})$

\begin{tabular}{|c|c|c|c|c|}
\hline & & \multicolumn{3}{|c|}{ Without preoperative preparation } \\
\hline \multicolumn{2}{|c|}{ Duration of jaundice } & 10 days & 2 weeks & 3-6 weeks \\
\hline \multirow[t]{4}{*}{ Rehberg-Tareev Test } & Daily diuresis (ml) & $\begin{array}{c}1276.7 \\
30.7 \\
1000.0 \\
1500.0\end{array}$ & $\begin{array}{c}1190.7 \\
50.6 \\
1000.0 \\
1300.0\end{array}$ & $\begin{array}{c}1137.0 \\
21.3 \\
1000.0 \\
1350.0\end{array}$ \\
\hline & $\begin{array}{l}\text { Minute urine output } \\
(\mathrm{ml} / \mathrm{min})\end{array}$ & $\begin{array}{l}0.88 \\
0.02 \\
0.69 \\
1.09\end{array}$ & $\begin{array}{l}0.83 \\
0.01 \\
0.69 \\
0.90\end{array}$ & $\begin{array}{l}0.79 \\
0.01 \\
0.69 \\
0.94\end{array}$ \\
\hline & $\begin{array}{l}\text { Glomerular filtration } \\
(\mathrm{ml} / \mathrm{min})\end{array}$ & $\begin{array}{c}100.9 \\
3.1 \\
78.2 \\
128.8\end{array}$ & $\begin{array}{c}88.6 \\
2.3 \\
77.0 \\
115.6\end{array}$ & $\begin{array}{c}73.7 \\
1.5 \\
60.0 \\
85.2\end{array}$ \\
\hline & Tubular reabsorption $(\%)$ & 99.0 & $\begin{array}{c}98.90 \\
0.03 \\
98.5 \\
99.0\end{array}$ & $\begin{array}{c}98.40 \\
0.07 \\
97.8 \\
99.0\end{array}$ \\
\hline
\end{tabular}

Note: $*$ - the difference between the indicators for the groups is significant $\mathrm{p}<0.01-0.001$

In the comparison group with the same duration of jaundice, a moderate decrease in diuresis was noted in $60 \%$ of patients, while a decrease in the glomerular filtration - only in $6.7 \%$ of patients, the tubular reabsorption was fully restored.

In 2-week jaundice, although the daily diuresis remained moderately reduced, it was within $1220-1340 \mathrm{ml}$ in $70 \%$ of patients, the glomerular filtration and the tubular reabsorption were restored. In the comparison group, diuresis is even more reduced, in $93.3 \%$ of patients it does not exceed $1000 \mathrm{ml}$ per day, the glomerular filtration was significantly reduced (table. 2,3 ) $-\mathrm{p}<0.01$, in $20 \%$ it was below the lower limit of the norm $(79.2 \mathrm{ml} / \mathrm{min})$. The others are within that boundary. 
Table 3 Renal function after the surgical treatment after preoperative preparation before discharge $(M \pm m ; R)$

\begin{tabular}{|c|c|c|c|c|}
\hline & & \multicolumn{3}{|c|}{ After preoperative preparation } \\
\hline \multicolumn{2}{|c|}{ Duration of jaundice } & 10 days & 2 weeks & 3-6 weeks \\
\hline \multirow[t]{4}{*}{$\begin{array}{l}\text { Rehberg-Tareev } \\
\text { Test }\end{array}$} & Daily diuresis $(\mathrm{ml})$ & $\begin{array}{l}1619,0^{*} \\
13.5 \\
1480.0 \\
1700.0\end{array}$ & $\begin{array}{l}1300.0 \\
16.3 \\
1220.0 \\
1400.0\end{array}$ & $\begin{array}{l}1334,0 * \\
18,2 \\
1200.0 \\
1500.0\end{array}$ \\
\hline & Minute urine output ( $\mathrm{ml} / \mathrm{min})$ & $\begin{array}{l}1.12 \\
0.03 \\
1.03 \\
1.18 \\
\end{array}$ & $\begin{array}{l}0.90 \\
0.07 \\
0.85 \\
0.97 \\
\end{array}$ & $\begin{array}{l}0.93 \\
0.01 \\
0.83 \\
1.04 \\
\end{array}$ \\
\hline & $\begin{array}{l}\text { Glomerular filtration } \\
(\mathrm{ml} / \mathrm{min})\end{array}$ & $\begin{array}{l}135,8^{*} \\
3.4 \\
100.0 \\
156.2\end{array}$ & $\begin{array}{l}118,6^{*} \\
4.3 \\
101.6 \\
171.7 \\
\end{array}$ & \begin{tabular}{l|}
75.2 \\
0.7 \\
68.8 \\
79.8 \\
\end{tabular} \\
\hline & Tubular reabsorption (\%) & 99.0 & 99.0 & $\begin{array}{l}98.60 \\
0.06 \\
98.0 \\
99.0\end{array}$ \\
\hline
\end{tabular}

Note: $*$ - the difference between the indicators for the groups is significant $\mathrm{p}<0.01-0.001$

In patients with jaundice lasting up to 3-6 weeks in the second group, the daily diuresis is the same as in two-week jaundice, but in comparison with the first group (without preliminary preparation), the diuresis is significantly higher $(\mathrm{p}<0.01)$. However, the glomerular filtration was below normal values $(68.8-78.6 \mathrm{ml} / \mathrm{min})$ in $60 \%$ of patients, the tubular reabsorption was reduced in $20 \%$ of patients. In the comparison group, a deeper impairment of the glomerular filtration was observed in $66.6 \%$ of patients, the half of them $(33.3 \%)$ was below $70 \mathrm{ml} / \mathrm{min}$ (60-69 $\mathrm{ml} / \mathrm{min})$. The tubular reabsorption was also impaired in $33.3 \%$ of patients. This indicates deep morphofunctional lesions of the kidneys with prolonged jaundice, especially in patients of the first group.

After surgical treatment before the discharge, the complete restoration of potassium-sodium balance in all media was noted in patients with 10-day jaundice. In patients with 2-week jaundice, hypokalemia and hyponatremia decreased from 50 to $18 \%$ or 2.8 times. Less significantly changed the intracellular balance. It's down from 90 to $60 \%$. At the same time, kaliuresis and sodium retention decreased from 65 to $46 \%$. In patients with 3-6-week jaundice, the extracellular hypokalemia persisted in $25 \%$ of cases, while the sodium content practically did not change. The intracellular potassiumsodium imbalance remained almost at the same level. The excretion of potassium in the urine decreased slightly from 100 to $85 \%$. In the first group, the potassium-sodium imbalance in $45 \%$ of patients and kaliuresis in $50 \%$ of patients with a corresponding percentage of sodium retention retained. In the second group, extracellular sodium was reduced in $80 \%$ of cases, hypokalemia remained in $35 \%$ of patients. The intracellular imbalance was $65 \%$. There was an increased excretion of potassium in the urine and sodium retention. Thus, the impairments of potassium-sodium balance are largely determined by the duration of obstructive jaundice. The preoperative 3-7 day preparation helps to stabilize the condition and improve outcomes after surgical treatment (table. 3). Surgical interventions without complex preoperative preparation even with 10-day jaundice contribute to the preservation of more than one-third of patients with the extracellular potassium-sodium imbalance and the increased potassium excretion in the urine, and this occurs especially in patients with 3-6 week jaundice, which should be taken into account during the rehabilitation in the near and remote postoperative periods.

After surgical treatment with preoperative preparation for 10-day jaundice, the restoration of daily diuresis is influenced by the normalization of sodium excretion in the urine and its content in plasma, the reduction of intoxication factors, such as erythropoiesis-stimulating agents (EPSA), alkaline phosphatase (ALP), the normalization of plasma potassium, fibrinogen and the erythrocyte aggregation ability and, especially, the normalization of bilirubin, the intracellular sodium and residual nitrogen, the reduction of potassium losses in the urine, the increase in intracellular potassium, and, consequently, the normalization of glomerular filtration and tubular reabsorption.

After emergency surgical treatment without preparation, an important role in maintaining daily diuresis is played by the content of urine potassium, sodium and plasma potassium, a moderate decrease in fibrinogen and erythrocyte aggregation, which indicates the preservation of microcirculatory disorders caused by a decrease in intoxication factors - bilirubin and residual nitrogen. The increased excretion of potassium in the urine and sodium retention remained. The role of alkaline phosphatase and especially the role of endogenous intoxication is reduced by the reducing of the absorption capacity of erythrocytes, the maintaining of dysproteinemia and the reducing of 
intracellular potassium. This impairs tubular reabsorption and glomerular filtration.

In the case of 3-6 weeks jaundice, the surgical treatment with preparation contributed to the normalization of sodium and potassium plasma, but the impact of the impaired aggregation of erythrocytes preserved, and this fact indicates the remaining microcirculation disorders, the increased intracellular sodium, normalized tubular reabsorption, the urinary potassium moderately decreased, intracellular potassium increased, diuresis although increased, but did not reach normal values (table. 3 ).

In surgical treatment without preparation, the negative effects of bilirubin and alkaline phosphotase, microcirculation disorders due to the high aggregation ability of erythrocytes remained, but the tubular reabsorption did not fully recover due to the increased potassium excretion in the urine and sodium retention, the glomerular filtration did not fully recover, daily diuresis was reduced (table 2).

\section{CONCLUSION}

The studies indicate the influence of the duration of jaundice on the severity of renal dysfunction. The effectiveness of preoperative preparation directly depends on the duration of cholestasis, contributing to the improvement of the course of the postoperative period with the restoration of the liver and kidney functions in patients with 10-day cholestasis and a significant improvement in these indicators with prolonged obstructive jaundice.

\section{REFERENCES}

[1] T.G. Bryukhovets, A.S. Pulikov, L.G. Levkovich, The Reaction of the Functional Glomerular System to the Effects of Toxic Substances, in: Actual Issues of Morphology, Collection of scientific, Krasnoyarsk, 2003, pp. 21-22.

[2] E.Z. Burnevich, E.V. Namestnikov, T.N. Lopatkina, Hepatorenal Syndrome. A Review of Literature, The Therapist. Archive 6 (2009) 76-80.

[3] L.S. Kizyukevich, Structural-Metabolic Reaction of Initial Nephron Departments on Elimination of Bile Hypertension in Animals with Experimental Cholestasis, Morpholog. Sheets 1-2 (2004) 13-15.
[4] C. Martinez-Prieto, M.C. Ortiz, L.A. Fortepiani et al., Haemodynamic and Renal Evolution of the Bile DuctLigated Rat, Clin. Sci. Colch. 98 (2003) 611-617.

[5] M.C. Ortiz, M.C. Manriquez, K.A. Nath et al., Vitamin E Prevents Renal Dysfunction Induced by Experimental Chronic Bile Duct Ligation, Kidney Int. 64 (2009) 950-961.

[6] M. Porst, A. Hartner, H. Krause et al., Inducible Nitric Oxide Synthase and Glomerular Hemodynamics in Rts with Liver Cirrhosis, Am. J. Physiol. Renal. Physiol. 281 (2008) 293-299.

[7] D.C. Tarng, T.P. Huang, T.Y. Liu et al., Effect of Vitamin E-bonded Membrane on the 8-hydroxy 2'deoxyguanosine Level in Leukocyte DNA of Hemodialysis Patients, Kidney Int. 58 (2000) 790-799.

[8] N. Nieto, S.L. Friedman, A.I. Cederbaum, Stimulation and Proliferation of Primary Rat Hepatic Stellate Cells by Cytochrome P450 2E1-derived Oxygen Species, Hepatology 35 (2002) 62-73.

[9] H. Jaeschke, G.J. Gores, A.I.Cederbaum et al., Mechanisms of Hepatotoxicity, Toxicol. Sci. 65 (2002) 166-176.

[10] Y.T. Huang, T.Y. Lee, H.C. Lin et al., Hemodynamic Effects of Salvia Miltiorrhiza on Cirrhotic Rats, Can. J. Physiol. Pharmacol. 79 (2001) 566-572.

[11] A. Cardenas, P. Gines, Hepatorenal Syndrome, Clin. Liver Dis. 10 (2006) 371-385.

[12] V. Arroyo, W. Jimenez, Complications of Cirrhosis. II. Renal and Circulatory Dysfunction. Lights and Shadows in an Important Clinical Problem, J. Hepatol. 32 (2000) 157-170.

[13] P. Gentilini, F. Vizzutti, A. Gentilini et al., Update on Ascites and Hepatorenal Syndrome, Dig. Liver Dis. 34 (2002) 592-605.

[14] F. Oberti, E. Vuillemin, J. Fort, P. Cales, Experimental Models of Portal Hypertension, Gastroenterol. Clin. Biol. 24 (2000) 896-901.

[15] A. Cardenas, P. Gines, Therapy Insight: Management of Hepatorenal Syndrome, Nat. Clin. Practice Gastroenterol. Hepatol. 3 (2006) 338-348.

[16] C.B. Herath, F.J. Warner, J.S. Lubel et al., Upregulation of Hepatic Angiotensin-Converting Enzyme 2 (ACE2) and Angiotensin-(1-7) Levels in Experimental Biliary Fibrosis, J. Hepatol. 47 (2007) 387-395. 